\title{
The Roll Stability Analysis of Semi-Trailer Based on the Wheel Force
}

\author{
Dong Wang ${ }^{1, *}$, Siwei Chen ${ }^{1}$, Weigong Zhang ${ }^{1}$ and Danjie Du ${ }^{2}$ \\ ${ }^{1}$ Collage of Instrumental Science and Engineering, Southeast University, Nanjing, 210096, China \\ ${ }^{2}$ North Carolina State University, Raleigh, 27695, USA \\ *Corresponding Author: Dong Wang. Email: kingeast16@seu.edu.cn \\ Received: 26 August 2021; Accepted: 27 September 2021
}

\begin{abstract}
It is different for the liquid tank semi-trailer to keep roll stability during turning or emergency voidance, and that may cause serious accidents. Although the scholars did lots of research about the roll stability of liquid tank semi-trailer in theory by calculating and simulation, how to make an effective early warning of rollover is still unsolved in practice. The reasons include the complex driving condition and the difficulty of the vehicle parameter obtaining. The feasible method used currently is evaluating the roll stability of a liquid tank semi-trailer by the lateral acceleration or the attitude of the vehicle. Unfortunately, the lateral acceleration is more useful for sideslip rather than rollover, and the attitude is a kind of posterior way, which means it is hard to take measures to cope with the rollover accident when the attitude exceeds the safety threshold. Considering the movement of the vehicle is totally caused by the wheel force, the rollover could also be predicted by the changing of the wheel force. Therefore, in this paper, we developed a method to analyze the roll stability by the vertical wheel force. A thorough experiment environment is established, and the effectiveness of the proposed method is verified in real driving conditions.
\end{abstract}

Keywords: Roll stability; wheel force; liquid tank semi-trailer; real driving conditions

\section{Introduction}

The liquid tank semi-trailer has become the main carrier for liquid chemical products transportation, because of the large transportation capacity and low transportation costs [1,2]. And it also brings serious safety risks for the road traffic. The report shows that the accident probability caused by the liquid tank semi-trailer accounts for about $80 \%$ of all dangerous goods transportation.

Like the ordinary heavy-duty semi-trailers, the liquid tank semi-trailer has a high center of gravity, a large load, a narrower track relative to the height of the vehicle, and a coupling effect between the tractor and the trailer [3]. All these factors lead to a quite low roll stability limit for the liquid tank semi-trailer. The literature shows nearly one-third accidents of heavy semi-trailers are rollover [4-6].

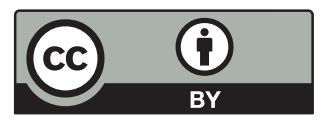

This work is licensed under a Creative Commons Attribution 4.0 International License, which permits unrestricted use, distribution, and reproduction in any medium, provided the original work is properly cited. 
What's worse is that the liquid in the tank has no definite shape and is fluid during driving $[7,8]$. That makes the trailer system is always in an unstable state and has the characteristics of low rollover threshold. In the actual transportation process, due to the limitation of vehicle axle load on the road transportation, the different density of liquid cargo, the restriction of thermal expansion, and contraction cause the liquid to not fill the entire tank, which means the trailer is essentially in partially filled condition $[9,10]$. In this situation, the liquid sloshing in the tank of the trailer has an obvious coupling effect with the dynamics of the whole vehicle [11]. Therefore, compared with ordinary heavy-duty semi-trailers, the roll stability of liquid tank semi-trailer is more difficult to be guaranteed, especially under driving conditions such as emergency braking, high-speed lane change or obstacle avoidance [12].

Therefore, to intervene the vehicle effectively before a rollover accident occurs, it is necessary to evaluate the roll stability of the liquid tank semi-trailer during driving by the vehicle dynamic parameters sensing. The rest of this study is organized as follows. Section 2 analyzes the dynamic model in the rollover processing and the proposed evaluation standard. Section 3 describes the design details of the vertical wheel force acquisition equipment, which is indeed a specially manufactured wheel force sensor. Section 4 introduces the test equipment for the actual vehicle experiment, including the distribution of the sensors and the protective equipment, and the experiment results of the real liquid tank semi-trailer are also shown in this section. At last, Section 5 concludes the paper.

\section{Roll Dynamics Model and the Evaluation Standard of Roll Stability}

\subsection{Roll Dynamics Model}

The roll dynamics model is essential to study the vehicle roll phenomenon. Considering that the roll of the trailer part is the main form of the vehicle roll, and the rear axle of the trailer is the dangerous point of the vehicle roll instability, the rear axle of the trailer is selected to establish the roll dynamics model in this paper, as shown in Fig. 1 [13], and the model could be also described by (1).

$$
\left(J_{2, x}+m_{2} h^{2}\right) \ddot{\phi}+d_{\dot{\phi}} \dot{\phi}+\left(c_{\phi}-m_{2} g h\right) \phi=m_{2} h a_{y, 1}
$$

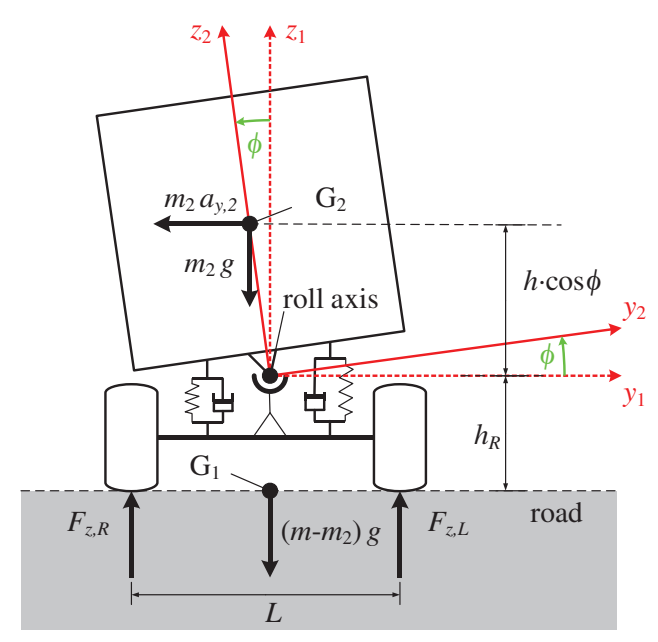

Figure 1: Roll dynamics model

The states in (1) are the roll angle $\phi$ and the lateral acceleration of the unsprung mass $a_{y, 1}$. Other parameters of the model, which could be obtained offline include the sprung mass roll moment of 
inertia $J_{2, x}$, the sprung mass $m_{2}$, the nominal height of $\mathrm{G} 2$ over roll axis $h$, the roll damping of passive suspension $d_{\dot{\phi}}$, and the roll stiffness of passive suspension $c_{\phi}$.

\subsection{Criterion of Roll Stability}

During the driving of the vehicle, even if there is a center of gravity deviation or lateral acceleration, as long as the road surface can provide sufficient support to balance the roll torque generated by the above situation, the vehicle will not roll over. A large number of simulations and experiments show that rollover accidents usually occur when the balance torque provided by the road surface is insufficient. Therefore, the rollover coefficient $C_{F}$ defined by the vertical load of the rear axle of the vehicle is the more accurate one among the currently recognized rollover standards, which is shown in (2).

$C_{F}=\frac{F_{z, L}-F_{z, R}}{F_{z, L}+F_{z, R}}$

Where $F_{z, L}$ and $F_{z, R}$ represent the vertical force on the left and right wheels, respectively. Obviously, the rollover coefficient $C_{F}$ satisfies $-1 \leq C_{F} \leq 1$, and when the vehicle is driving normally on a level road, since $F_{z, L}$ and $F_{z, R}$ are the same, $C_{F}$ is equal to 0 . At the same time, if $F_{z, L}$ does not equal to $F_{z, R}$, the vehicle has a rollover risk. In extreme cases, if $F_{z, L}=0$ or $F_{z, R}=0$, then the left or right wheel lifts off, which indicates that a rollover is about to occur.

Because the vertical wheel forces are hard to be obtained in real time, they are often estimated by using the roll dynamics model shown in Fig. 1. And at this time, Eq. (2) could be written in the form of (2). Where $m$ is the overall vehicle mass, $T$ is the axle length, $h_{R}$ is the height of the rolling axis from the ground, and $a_{y, 2}$ is the lateral acceleration of the sprung mass.

$C_{F}=\frac{2 m_{2}}{m T}\left(\left(h_{R}+h \cos \phi\right) \frac{a_{y, 2}}{g}+h \sin \phi\right)$

Furthermore, Assuming $m_{2} \gg\left(m-m_{2}\right),\left(h_{R}+h \cos \phi\right) a_{y, 2} / g \gg h \sin \phi$, and the roll angle $\phi$ to be small, Eq. (3) is approximated by (4), in which $h_{R}$ and $h$ could be measured at the start of each ride, and $a_{y, 2}$ also could be given by an IMU. Thus, Eq. (3) is available for ordinary vehicles.

$C_{F} \approx \frac{2\left(h_{R}+h\right) a_{y, 2}}{T g}$

Unfortunately, for the liquid tank semi-trailer, the liquid in the tank might shake during the driving condition, which causes the position of the center of gravity of the tank to change. That means the parameter $h$ is always changing [14-15]. In this situation, the estimation of $C_{F}$ could not follow (4). To solve this problem and then evaluate the roll stability of the liquid tank semi-trailer accurately, we should come back to (2) and sensor the wheel force in real time.

\section{Design of the Wheel Force Sensor}

\subsection{Coordinates Definition}

The wheel force sensor could measure various forces between the wheel and the road and of course, it has to be installed on the wheel itself. Since the wheel is rotating during driving, there is a difference between the sensing coordinate and the calculating coordinate of the wheel force. To deal with this problem, two coordinates are defined, respectively in Fig. 2. 


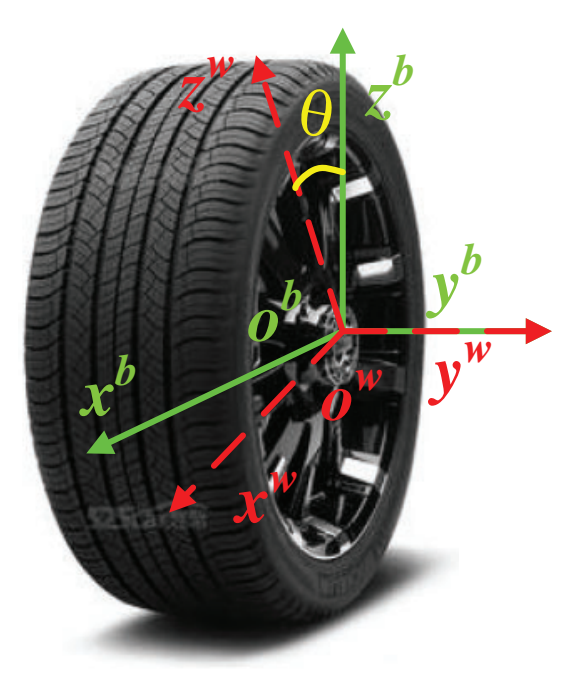

Figure 2: Definition of the coordinates

In Fig. 2, the vehicle body coordinate $\left\{o^{b} x^{b} y^{b} z^{b}\right\}$ (wheel force calculating coordinate) is relatively stationary with the body of the trailer with $o^{b}$ on the centre of the wheel, $o^{b} z^{b}$ perpendicular to the ground, $o^{b} x^{b}$ to the front of the trailer body, and $o^{b} y^{b}$ to the side. In addition, the origin of the wheel coordinate $\left\{o^{w} x^{w} y^{w} z^{w}\right\}$ (wheel force sensing coordinate) coincides with that of $\left\{o^{b} x^{b} y^{b} z^{b}\right\}$, and is fixed on the wheel.

It is worth to note that these two coordinates completely coincide at the beginning, then as the wheel rotates, the coordinate $\left\{o^{w} x^{w} y^{w} z^{w}\right\}$ rotates around the $o^{b} x^{b}$ axis relative to $\left\{o^{b} x^{b} y^{b} z^{b}\right\}$. At this time, the conversion relationship between two coordinates could be described in (5).

$\left[\begin{array}{l}x^{b} \\ y^{b} \\ z^{b}\end{array}\right]=\left[\begin{array}{ccc}\cos \theta & 0 & \sin \theta \\ 0 & 1 & 0 \\ -\sin \theta & 0 & \cos \theta\end{array}\right]\left[\begin{array}{l}x^{w} \\ y^{w} \\ z^{w}\end{array}\right]$

\subsection{Sensor Design}

In the scene that this paper is concerned about, the measurement of vertical force is essential [16]. However, due to the rotation of the wheel, the vertical and longitudinal forces are coupled with each other in the sensing process, and it can be seen from (5) that the relationship between the measured wheel forces in the wheel coordinate $\left\{o^{w} x^{w} y^{w} z^{w}\right\}$ and the actual wheel forces in the wheel coordinate $\left\{o^{b} x^{b} y^{b} z^{b}\right\}$ obeys (6). Where $\left[F_{x}^{b}, F_{y}^{b}, F_{z}^{b}\right]$ and $\left[F_{x}^{w}, F_{y}^{w}, F_{z}^{w}\right]$ are the actual and measured vertical, side and longitudinal wheel forces, respectively.

$$
\left\{\begin{array}{l}
F_{x}^{b}=\cos \theta \cdot F_{x}^{w}+\sin \theta \cdot F_{z}^{w} \\
F_{y}^{b}=F_{y}^{w} \\
F_{z}^{b}=-\sin \theta \cdot F_{x}^{w}+\cos \theta \cdot F_{z}^{w}
\end{array}\right.
$$

In order to sense both vertical and longitudinal wheel forces, a spoke elastic body is designed as in Fig. 3 [17]. The inner ring of the elastic body is connected to the axle of the trailer, and the outer ring is connected to the wheel. When the wheel is under force, the eight strain beams located between 
the inner and the outer ring will be slightly deformed, and these deformations can be converted into electrical signals by the bridge composed of resistance strain gauges [18].

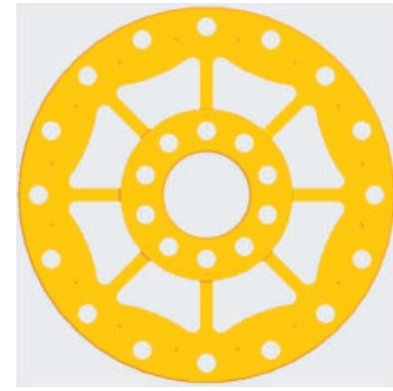

(a)

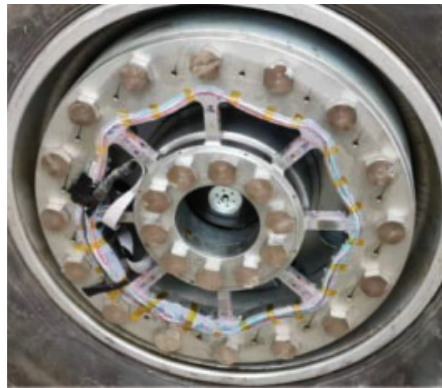

(b)

Figure 3: Elastic body: (a) Design diagram; (b) Physical photo

The division of strain gauges on the strain beams is shown in Fig. 4 [19]. Specifically, the strain gauges, which are used to sense $F_{x}^{w}$ and $F_{z}^{w}$ are located in the middle of the strain beams to sensitive tension and pressure on it [20]. And the strain gauges on the bottom of the beam are sensitive to the shear force for the measurement of $F_{v}^{w}$. Furthermore, to improve the sensitivity and reduce the common mode error, the strain gauges form differential bridges as in Fig. 5 [21]. By the elastic body and these bridges, the forces in $\left\{o^{w} x^{w} y^{w} z^{w}\right\}$ could be converted into the voltage signal and then be collected and processed to obtain the actual wheel forces in $\left\{o^{b} x^{b} y^{b} z^{b}\right\}$. In this course, it's worth noting that the angle $\theta$ is also a need to finish the coordinate system conversion based on (6). Therefore, an encoder which could record the rotational angle of the wheel is indispensable in the wheel force sensor assembly.
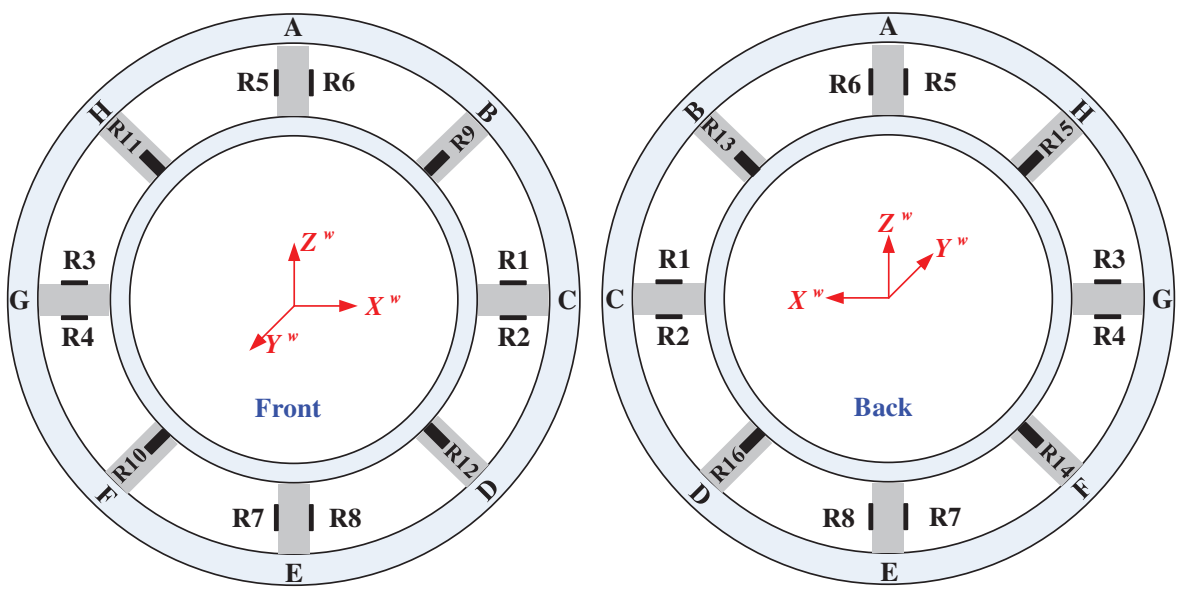

Figure 4: Division of strain gauges

Fig. 6 shows the complete wheel force sensor assembly [22]. The elastic body (4) connects the axle and the rim (2) by the inner and outer ring flanges (1) and (3), and in order not to interfere with the axle head, the special flanges are relatively long. The elastic body and the strain gauges on it are protected by the metal cover (5) to keep the water and dust away. The encoder and the signal acquisition circuit are located in the circuit box (6). The fastener (ㄱ) is used to fix one end of the encoder to the trailer body, so that the output of the encoder could directly reflect the rotation angle of the wheel. 

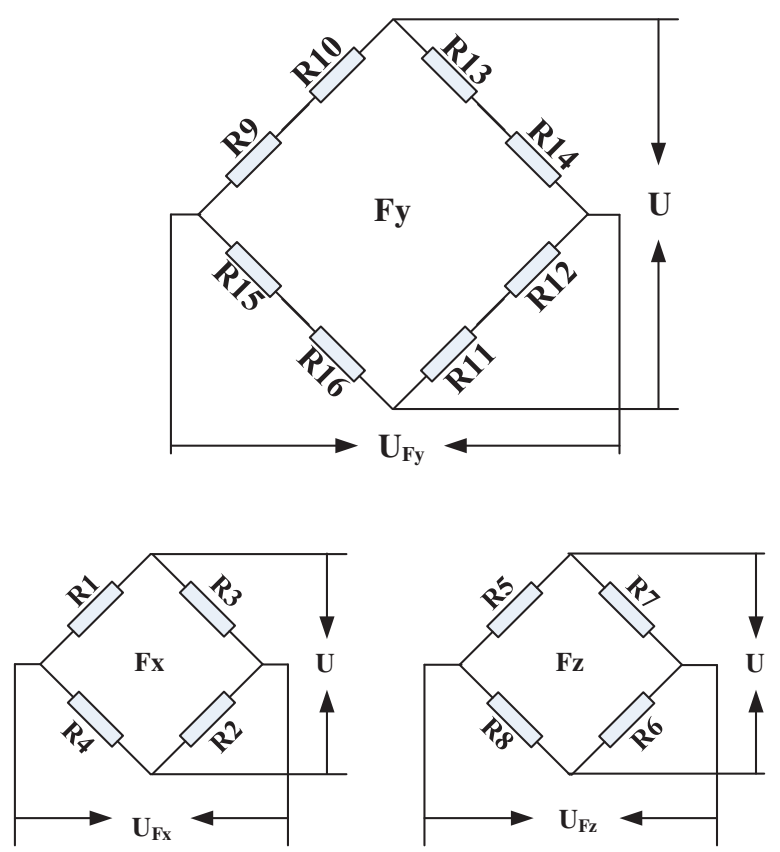

Figure 5: Differential bridge

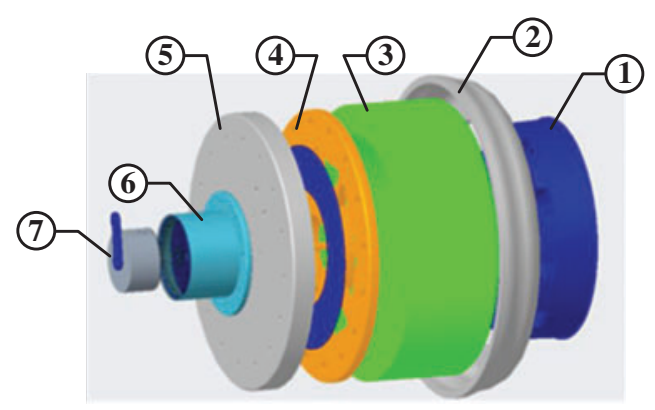

(a)

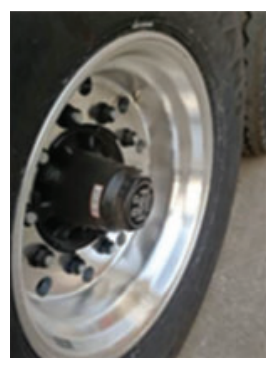

(b)

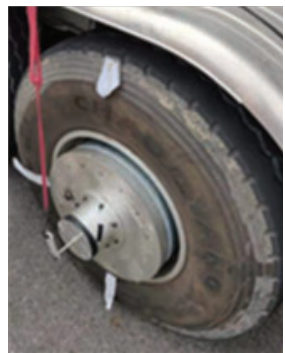

(c)

Figure 6: Wheel force sensor assembly: (a) Exploded view of the wheel force sensor assembly; (b) Axle head; (c) Physical photo

The wheel force information collected by the wheel force sensor will be wirelessly transmitted to the processing terminal on the trailer via Bluetooth, and decoupling calculations will be performed to 
obtain the actual wheel force. As in Figs. 7 and 8, the raw data obtained directly by the wheel force sensor shows an oscillating waveform due to the rotation of the wheel, and the oscillation amplitudes of $F_{x}^{w}$ and $F_{z}^{w}$ are equal, while the phase difference is $\pi / 2$. After decoupling with the rotation angle, the actual wheel forces that can be used for the analysis of the roll stability of the liquid tank semi-trailer are as shown in Fig. 7. It can be seen from Fig. 8 that the vertical wheel force remains unchanged, and the longitudinal wheel force is near 0 , which indicates that the trailer is maintaining a straight-line state at a constant speed.
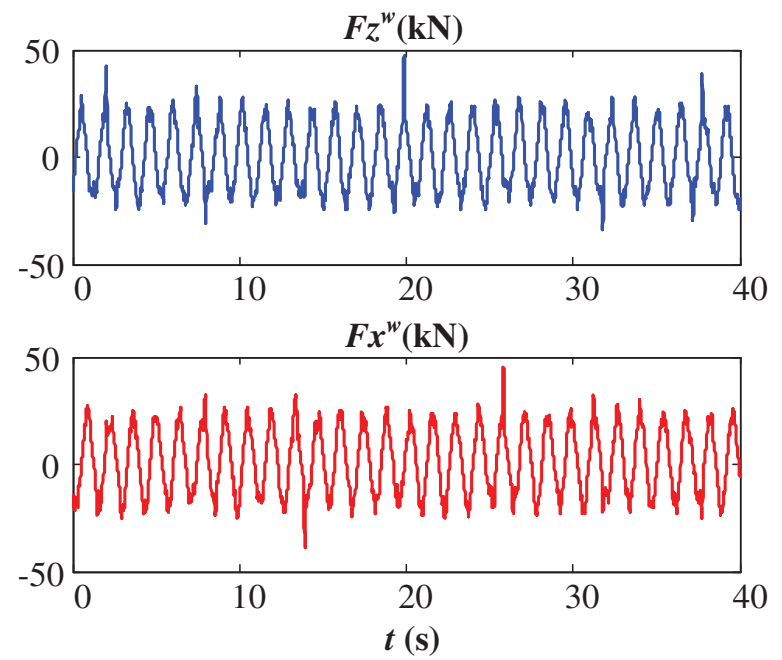

Figure 7: Raw wheel forces in the wheel coordinate

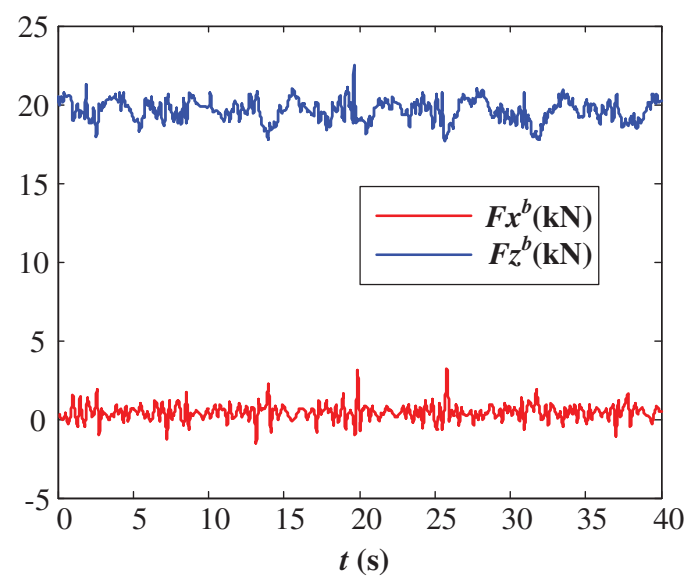

Figure 8: Decoupling wheel forces in the trailer body coordinate

\section{Real Vehicle Experiment}

\subsection{Experimental Equipment}

In order to test the actual effect of the trailer rollover stability analysis method used in this paper, on the premise of ensuring the safety of personnel, a liquid tank semi-trailer is modified according to the method shown in Fig. 9. 


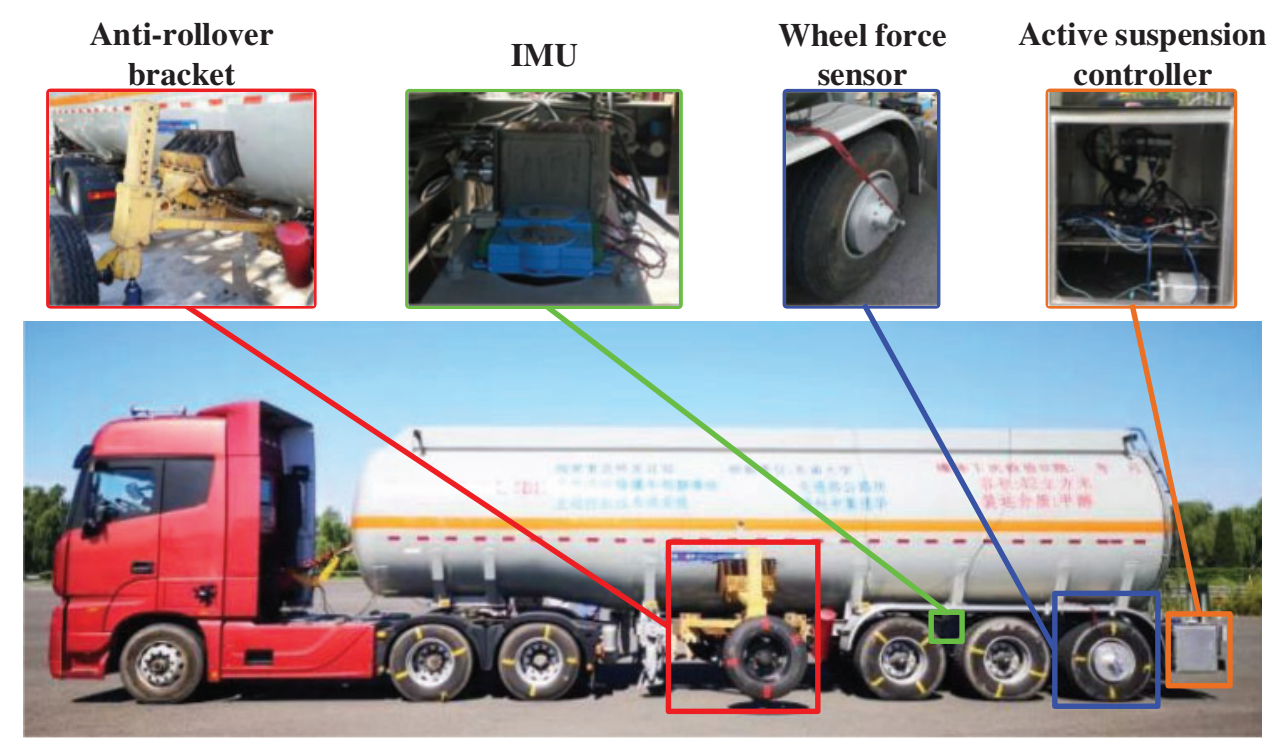

Figure 9: Liquid tank semi-trailer for experiment

First of all, an anti-rollover bracket is fixed on both sides of the tank. This bracket could provide effective support when the trailer is about to roll over, thereby avoiding accidents and ensuring the safety of the driver. Then the active suspension is equipped to adjust the pressure of the hydraulic lever, so that the trailer can regain its balance when a roll is about to occur. This active suspension can reduce the risk of rolling effectively, and its control signal could also be used to verify the analysis results of rollover stability based on wheel force. At last, two sets of sensors are installed to obtain the driving information of the semi-trailer. One is the high-precision inertial measurement unit (IMU), and the other is the wheel force sensor. The IMU is put on the middle of the trailer and is used to sense the three-axis acceleration and angular velocity. The Wheel force sensors are installed on the last axle of the trailer to measure the longitudinal and vertical wheel forces on the left and right sides, since the research shows that the last axle is where the rollover often occurs.

\subsection{Experimental Results}

\subsubsection{Half-Load Steady State Rotation Test}

The half-load steady state rotation test, which is widely used in the roll stability analysis, is carried out to compare the three stability criteria of wheel force, lateral acceleration, and roll angle. To ensure safety, the professional vehicle test site and experienced drivers are selected to carry out the dangerous test. The trailer trajectory of the test which consists of three intact left and right turns, is shown in the Fig. 10.

The wheel forces and vehicle speed in the half-load steady state rotation test are shown in Fig. 11. It can be clearly seen from the figure that the trend of wheel force is basically the same as the trailer speed. When the trailer is driving in a straight line, the vertical forces on the left and right wheel are almost the same, since the trailer is in balance. However, when the trailer begins to turn, the pressure increases in the turning direction and decreases in the opposite. Thus, the vertical forces on both sides change reversely. 
CMC, 2022, vol.71, no.1

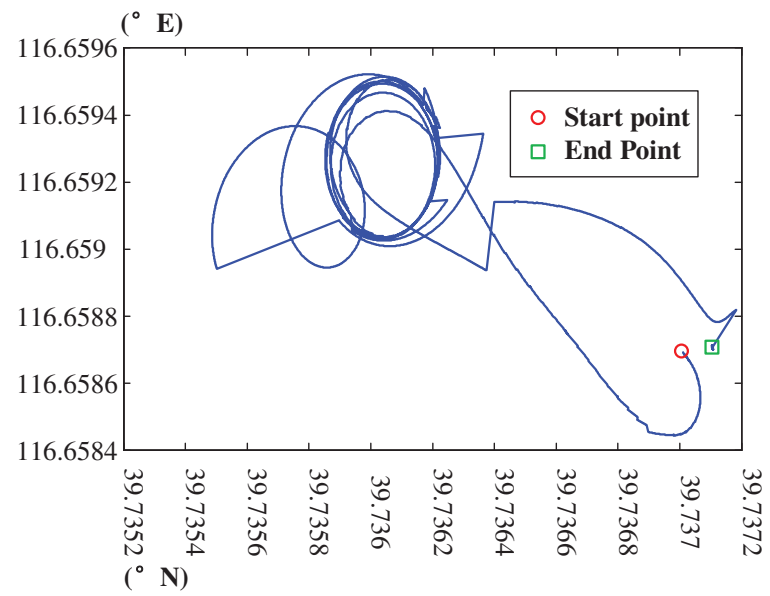

Figure 10: Trajectory of the half-load steady state rotation test
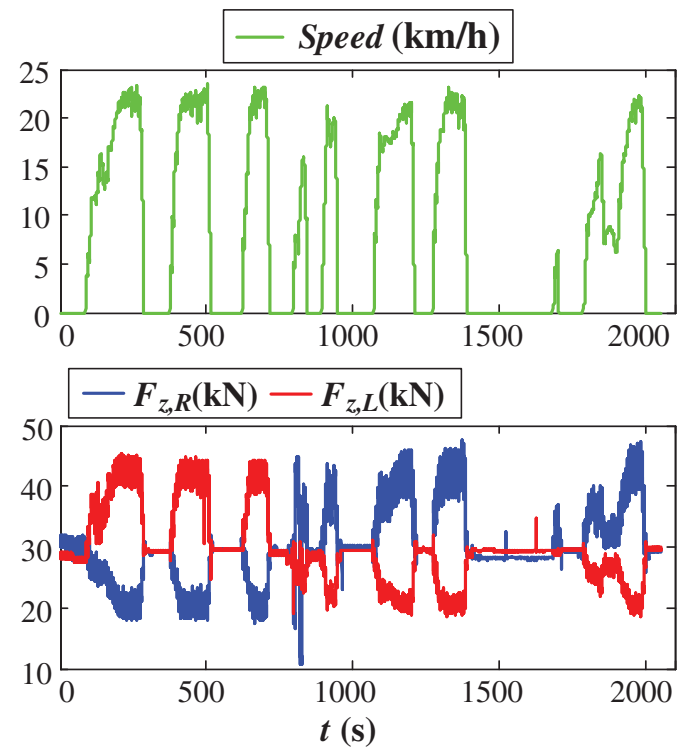

Figure 11: Data of the half-load steady state rotation test

The comparison of the three roll stability criteria including wheel force, lateral acceleration, and roll angle are in Fig. 12. What can be noticed is that the three criteria have the same general trends but different details. In the zoomed-in figure, it could be found that when the trailer has a rollover risk, the first thing that is sensitive to this change is $C_{F}$, which is calculated by the vertical wheel force, since the changing of the wheel loads is the reason of the changing of the attitude. Considering the situation of the whole six left and right turns, using $C_{F}$ as the criterion can buy one more second for the active control of the trailer, which is very critical for driving safety under extreme conditions. The half-load steady state rotation test has a low risk factor, and the most intense maneuver occurred at the end of the second left turn, which is with the deceleration of $0.52 \mathrm{~m} / \mathrm{s}^{2}$, so that the active suspension did not work during the test. 


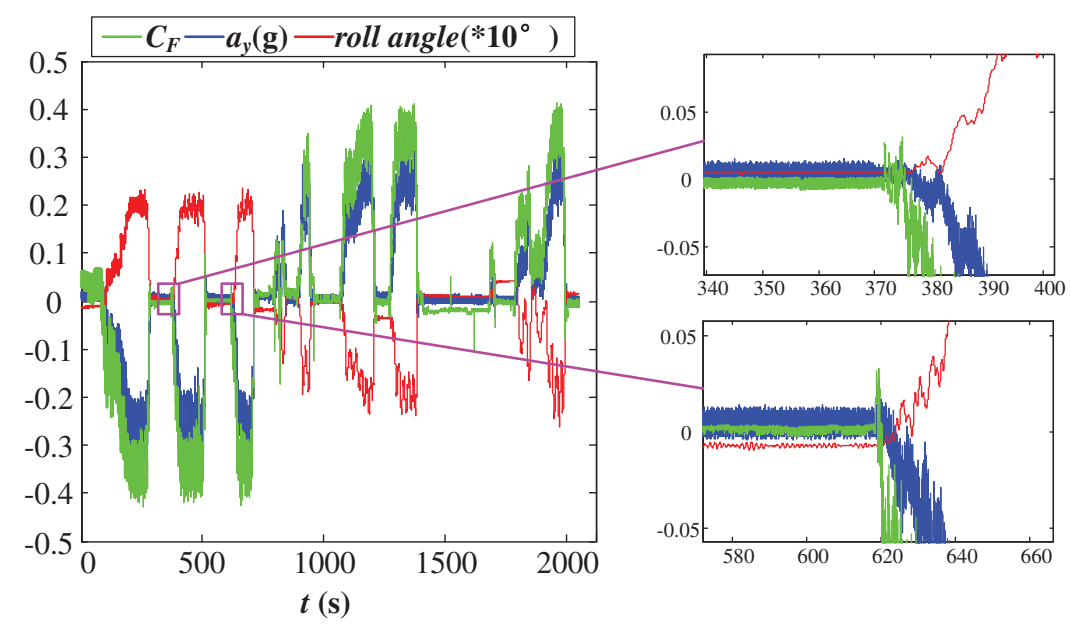

Figure 12: Comparison of the three roll stability criteria in half-load steady state rotation test

\subsubsection{Steering Brake Test}

The steering brake test is highly dangerous since the speed of the trailer is higher, and the brake pedal needs to be depressed while turning [23]. The changing of the speed and the situation of the EBS signal of the active suspension (20 means on and 0 means off) in the steering brake test is shown in Fig. 13. It is obviously that there are three severe decelerations in this test, and the decrease speed are $1.32 \mathrm{~m} / \mathrm{s}^{2}$ (around the 50th second, decreased by $11.4 \mathrm{~km} / \mathrm{h}$ in $2.4 \mathrm{~s}$ ), $1.50 \mathrm{~m} / \mathrm{s}^{2}$ (around the 150 th second, decreased by $11.9 \mathrm{~km} / \mathrm{h}$ in $2.2 \mathrm{~s}$ ) and $1.49 \mathrm{~m} / \mathrm{s}^{2}$ (around the 275 th second, decreased by 12.9 $\mathrm{km} / \mathrm{h}$ in $2.4 \mathrm{~s}$ ) respectively. These three decelerations have all triggered the active suspension controller. Furthermore, Fig. 14 shows that the response sensitivity of the $C_{F}$ criterion is still better than the lateral acceleration and roll angle under emergency braking conditions.

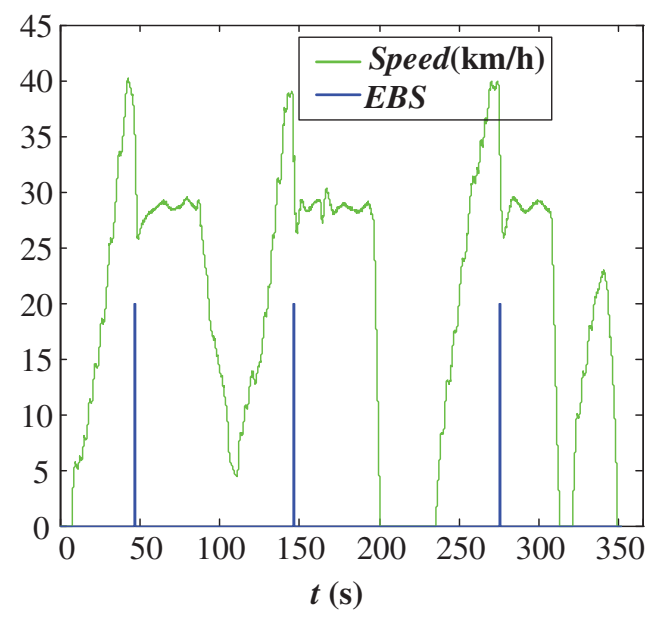

Figure 13: Data of the steering brake test 


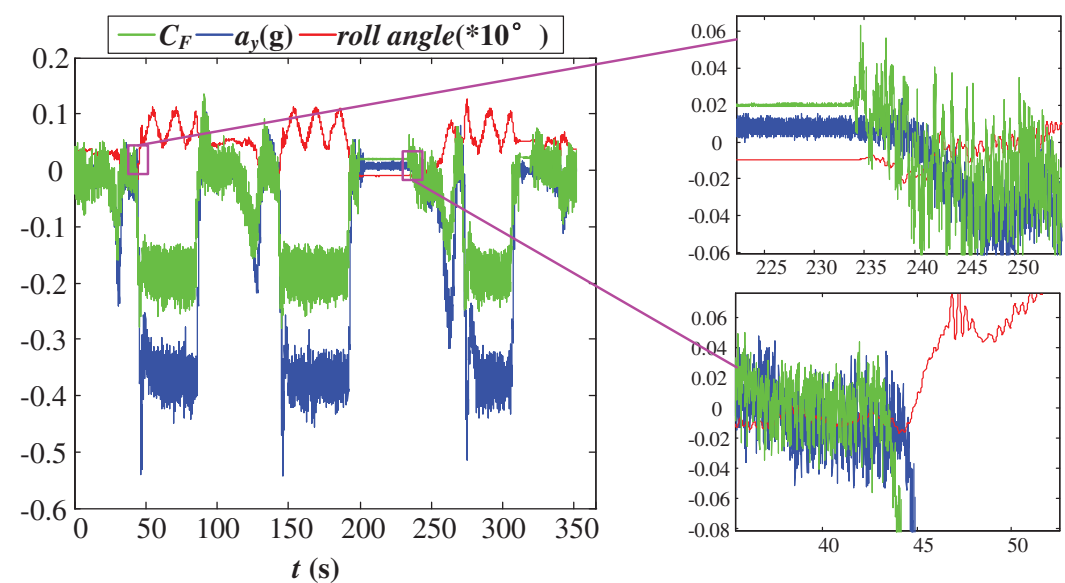

Figure 14: Comparison of the three roll stability criteria in steering brake test

\section{Conclusion}

The rollover stability analysis is crucial for the liquid tank semi-trailer to guarantee the safety of the driver and the equipment. Compare with the lateral acceleration and roll angle, the rollover coefficient $C_{F}$ defined by the vertical load is more credible based on the relative research. While the $C_{F}$ here could not be evaluated by the approximate formula, since when the tank is half loaded, its center of gravity will change in real time during driving. Thus a special wheel force sensor is designed in this paper. Then the half-load steady state rotation and steering brake tests are carried out to verify that the $C_{F}$ calculated by the vertical wheel forces is more sensitive than the other commonly used stability criteria. Therefore, that the suspension system can take action as early as possible to avoid rollover accidents.

Acknowledgement: This work was supported by the Suzhou Key industrial technology innovation project SYG202031.

Funding Statement: This work was supported by the Suzhou Key industrial technology innovation project SYG202031.

Conflicts of Interest: The authors declare that they have no conflicts of interest to report regarding the present study.

\section{References}

[1] Y. Q. Sun, C. Cole and M. Spiryagin, "Monitoring vertical wheel-rail contact forces based on freight wagon inverse modelling," Advances in Mechanical Engineering, vol. 7, no. 5, pp. 1-11, 2015.

[2] G. Xia, M. Zhao, X. Tang, S. Wang and L. Zhao, "Linear reversing control of semi-trailer trains based on hitch angle stable and feasible domain," Control Engineering Practice, vol. 104, no. 1, pp. 104625, 2020.

[3] S. Q. Zhang, H. Sun, Y. F. Zhang and Y. Liu, "Analyze and control on the flexible body of tractor semi-trailer rollover," in Int.Conf. on Mechanics Design, Manufacturing and Automation (MDM), Suzhou, ChinaHINA, pp. 607-611, 2017.

[4] C. Cheng and D. Cebon, "Improving roll stability of articulated heavy vehicles using active semi-trailer steering," Vehicle System Dynamics, vol. 46, no. sup1, pp. 373-388, 2008.

[5] D. Han, B. Shen, W. Zhou, X. Zhang and Q. Li, "The study of tanker semi-trailer's steering stability on the fluid-structure interaction," Procedia Environmental Sciences, vol. 12, no. 2, pp. 1082-1088, 2009. 
[6] Z. Zhao, M. Feng, G. Peng, J. Liu and Y. Tao, "Simulation and analysis on rollover stability of heavy semi-trailer train based on Trucksim," in 3rd Int. Conf. on Intelligent Green Building and Smart Grid, Yilan, Taiwan, China, Natl Ilan Univ, pp. 1-4, 2018.

[7] J. Semetko, I. Janosko and P. Pernis, "Increase of driving pulls of the tractor by trailer," in 31st Int. Symp. on Agricultural Engineering, Opatija, Croatia, pp. 59-64, 2003.

[8] S. Zhou, S. Zhang, G. Zhao and C. Tang, "Lateral stability control of car-trailer combination based on 4WS," in 2010 Int. Conf. on Measuring Technology and Mechatronics Automation, Changsha, China, vol. 2, pp. 576-579, 2010.

[9] W. Sun, X. Zhang, S. Shi and X. He, "Vehicle classification approach based on the combined texture and shape features with a compressive DL," IET Intelligent Transport Systems, vol. 13, no. 7, pp. 1069-1077, 2019.

[10] X. Wang and Q. Wang, "Application of dynamic programming algorithm based on model predictive control in hybrid electric vehicle control strategy," Journal of Internet of Things, vol. 2, no. 2, pp. 81-87, 2020.

[11] D. Wu, Y. Liu, Z. Xu and W. Shang, "Design and development of unmanned surface vehicle for meteorological monitoring," Intelligent Automation \& Soft Computing, vol. 26, no. 5, pp. 1123-1138, 2020.

[12] H. Wang, Q. Xue, T. Cui, Y. Li and H. Zeng, "Cold start problem of vehicle model recognition under cross-scenario based on transfer learning," Computers Materials \& Continua, vol. 63, no. 1, pp. 337-351, 2020.

[13] D. Odenthal, T. Bünte and J. Ackermann, "Nonlinear steering and braking control for vehicle rollover avoidance," in 1999 European Control Conf., Karlsruhe, Germany, pp. 598-603, 1999.

[14] J. Wang, T. Zhang, B. Jin and S. Wu, "A novel sins/iusbl integration navigation strategy for underwater vehicles," Journal of Cyber Security, vol. 1, no. 1, pp. 1-10, 2019.

[15] L. Zhu, Y. Wang, L. Wang and Z. Wu, "Stability analysis of transfer alignment filter based on the m theory," Computers Materials \& Continua, vol. 59, no. 3, pp. 1015-1026, 2019.

[16] X. Zhang, X. Yu, W. Sun and A. Song, "An optimized model for the local compression deformation of soft tissue," KSII Transactions on Internet and Information Systems, vol. 14, no. 2, pp. 671-686, 2020.

[17] M. Kieninger, A. Rupp and K. Rueffer, "A new wheel force transducer for the investigation of loads on rail bound vehicles in the urban traffic," Materialprufung, vol. 50, no. 1-2, pp. 42-50, 2008.

[18] X. Zhang, H. Wu, W. Sun, A. Song and S. K. Jha, "A fast and accurate vascular tissue simulation model based on point primitive method," Intelligent Automation and Soft Computing, vol. 27, no. 3, pp. 873-889, 2021.

[19] D. Wang, S. Chen, X. Li, W. Zhang and H. Jin, "Research on rotational angle measurement for the smart wheel force sensor," Sensors, vol. 20, no. 4, pp. 1037, 2020.

[20] D. Fujiwara, T. Oshima and K. Iizuka, "Measuring the normal stress distribution acting on a locked-wheel of push-pull locomotion rovers via a wheel sensor system," Sensors (Switzerland), vol. 20, no. 16, pp. 1-22, 2020.

[21] D. D. Falco, G. D. Massa, S. Pagano and S. Strano, "Wheel force transducer for shimmy investigation," Lateral, vol. 2218, pp. 1207-1212, 2015.

[22] D. Wang, G. Lin, W. Zhang and T. Jiang, "Angle error compensation in wheel force transducer," Measurement, vol. 77, no. 2, pp. 203-212, 2016.

[23] A. M. C. Odhams, R. L. Roebuck, B. A. Jujnovich and D. Cebon, "Active steering of a tractor-semi-trailer," Proceedings of the Institution of Mechanical Engineers, Part D: Journal of Automobile Engineering, vol. 225, no. 7, pp. 847-869, 2011. 